\title{
Increased mortality among people with anxiety disorders: total population study
}

\author{
Sandra M. Meier, Manuel Mattheisen, Ole Mors, Preben B. Mortensen, Thomas M. Laursen
} and Brenda W. Penninx

\section{Background}

Anxiety disorders and depression are the most common mental disorders worldwide and have a striking impact on global disease burden. Although depression has consistently been found to increase mortality; the role of anxiety disorders in predicting mortality risk is unclear.

\section{Aims}

To assess mortality risk in people with anxiety disorders.

\section{Method}

We used nationwide Danish register data to conduct a prospective cohort study with over 30 million person-years of follow-up.

\section{Results}

In total, 1066 (2.1\%) people with anxiety disorders died during an average follow-up of 9.7 years. The risk of death by natural and unnatural causes was significantly higher among individuals with anxiety disorders (natural mortality rate ratio $(\mathrm{MRR})=1.39,95 \% \mathrm{Cl} 1.28-1.51$; unnatural $\mathrm{MRR}=2.46,95 \% \mathrm{Cl} 2.20-2.73$ ) compared with the general population. Of those who died from unnatural causes, 16.5\% had comorbid diagnoses of depression (MRR $=11.72,95 \% \mathrm{Cl}$ 10.11-13.51).

\section{Conclusions}

Anxiety disorders significantly increased mortality risk. comorbidity of anxiety disorders and depression played an important part in the increased mortality.

\section{Declaration of interest}

None.

\section{Copyright and usage}

(c) The Royal college of Psychiatrists 2016.
Anxiety disorders and depression are among the most common mental disorders worldwide. ${ }^{1}$ Together they account for over $50 \%$ of the global disease burden in terms of disability-adjusted life years attributable to mental health disorders and substance use. $^{2}$ The association between depression and mortality is now well established, ${ }^{3-11}$ particularly higher rates of suicide have been reported among people diagnosed with depression, ${ }^{3,12}$ but the excess mortality risk extends to natural causes. ${ }^{13}$ In contrast, data on mortality risk in people with anxiety disorders are conflicting, providing more equivocal results of an absent, ${ }^{10,14}$ favourable, ${ }^{5,15}$ unfavourable $^{16-18}$ or even $\mathrm{U}$ shape relationship. ${ }^{6}$ These conflicting data might be because of the heterogeneity in sample size, gender, anxiety subtypes, somatic comorbidities ${ }^{8,11}$ and causes of death studied. Further, only a few studies have assessed mortality risk among patients with different specific anxiety disorders. ${ }^{14,17}$ Large prospective studies taking into account such factors are thus still missing in this area. In order to gain better insight in the mortality risk among patients with (specific) anxiety disorders we conducted a detailed population-based study using nationwide Danish register data.

We comprehensively assessed mortality rates across varying causes of death among people diagnosed with anxiety disorders. Given the clinical heterogeneity of anxiety diagnoses, we calculated mortality rates across the spectrum of specific anxiety disorders. As anxiety disorders are highly comorbid with depression ${ }^{1}$, we were specifically interested in independent and synergistic effects of these disorders on mortality risk. We therefore assessed whether mortality rates were elevated in individuals with anxiety disorders independent of comorbid depression and whether patients with both diagnoses were at an especially enhanced risk of dying. As a last step we assessed potential mediating effects of somatic comorbidity and substance misuse on mortality risks. A better understanding of excess mortality - and its causes - among patients with anxiety disorders could help to prevent these deaths.

\section{Method}

\section{Study setting}

We conducted a nationwide follow-up study. The main exposure variables were defined as a first psychiatric in-patient or outpatient contact for anxiety disorders. The outcome variable was defined as death by any natural cause (diseases and medical conditions) or unnatural cause (suicide, accidents and homicide) within the follow-up period. Data were collected on the cohort of people born in Denmark between 1 January 1955 and 31 November 2006. The follow-up time started on their first birthday or 1 January 2002, whichever came later, in order to ensure complete information on cohort members. Follow-up ended at date of emigration from Denmark, date of death or 31 December 2011, whichever came first. This provided a maximum follow-up period of 10 years and a maximum age of 57 for the cohort members. We focused on deaths among younger age groups of the population in order to reduce probable biases because of selective survival. Data on cohort members were collected through linkage of four Danish population registers (see online supplement DS1). All residents of Denmark, including immigrants, have a unique personal identification (CRS) number that is used in all national registers, which enables data to be linked across these registers. The study was approved by the Danish Data Protection Agency. All personal information from the registers is anonymised when used for research purposes, and by Danish law, informed consent is not needed for register-based studies.

\section{Exposure variables}

The main exposure variables were defined as treatment for anxiety disorder. Using the Danish Psychiatric Central Register ${ }^{19}$ and the Danish National Patient Register ${ }^{20}$ we identified all patients diagnosed with anxiety disorders (ICD-10: F40.00-F40.20, 
F41.00-F41.10, F42.00-F43.10). ${ }^{21}$ Date of onset was defined as the day of the first contact for the diagnosis in question.

\section{Outcome variables}

The outcome of interest was time to death. The date and cause of death were identified from the Danish Register of Causes of Death. ${ }^{22}$ All-cause mortality was defined as ICD-10 codes A00Y98 and categorised into the following groups: deaths from diseases and medical conditions (natural death) ICD 10: A00R99, and deaths from external causes (unnatural death) ICD-10: V01-Y98. Unnatural causes of death comprised suicides (ICD-10: X60-X84, Y10-Y34), homicides (ICD-10: X85-Y09, Y87.1) and accidents (ICD-10: V01-X59, Y10-Y86, Y87.2). Natural causes comprised death by cancer (ICD-10: C00-D48), cardiac diseases (ICD-10: I00-I99), respiratory diseases (ICD-10: J00-J99), digestive conditions (ICD-10: K00-K93) and remaining causes.

\section{Covariates}

Information on age, gender, calendar period, maternal and paternal age at time of birth, and place of residence was obtained from the Danish Civil Registration System. ${ }^{23}$ Somatic comorbidities were assessed using the Charlson Comorbidity Index (CCI), which is based on 19 severe chronic diseases weighted from one to six corresponding to the severity of the disease. ${ }^{24}$ Information on substance misuse (ICD- $8^{25}$ code 291.xx, 303.xx, 304.xx, 571.09, 571.1x and ICD-10 code F10-F16, F18, F19, I85, K70) and depression (ICD-8 code: 296.x9, 298.09, 298.19, 300.49, 301.19; ICD-10 code: F32.00-F33.99, F34.10-F34.90, F38.00-F39.99) was retrieved from the Danish Psychiatric Central Register ${ }^{19}$ and the Danish National Patient Register. ${ }^{20}$

\section{Sibling control studies}

To account for possible familial confounding, we performed additional analyses that used unaffected full siblings of patients as controls. In this analysis, we identified as cases those individuals with anxiety disorders who also had full siblings without anxiety disorders, and these individuals were compared with their unaffected full siblings using matched conditional Cox regression.

\section{Data analyses}

Mortality rate ratios (MRRs) were calculated for natural and unnatural causes of death as well as all causes. We fitted three statistical models to these outcomes, using the log-linear Poisson regression with the GENMOD procedure in SAS, version 9.3. In the first model we adjusted for demographic characteristics, in the second model somatic comorbidity, which was defined as a CCI of $0,1,2,3$ or more, and in the third model effects were adjusted for depression. Of special interest was an evaluation of whether the MRRs varied between lifetime diagnoses of anxiety disorders only, depression only or both disorders by testing for statistical interaction. As anxiety disorders and depression are also frequently comorbid with substance misuse, ${ }^{26}$ we additionally adjusted results for comorbid substance misuse. Finally, we studied potential mediating effects of somatic comorbidity and substance misuse on mortality risk. In our sensitivity analyses, we assessed differences in the psychiatry-mortality associations by gender and time since diagnosis. A more detailed description can be found in online supplement DS1.

\section{Results}

From 1 January 2002 to 31 December 2011 a total of 3270650 people were included in the study cohort contributing 31213252 person-years at risk. In 51373 (1.6\%) cohort, members follow-up ended before the end of the study; 50521 emigrated from Denmark and 852 were lost to follow-up. The mean follow-up time for the cohort members was 9.7 years. In total, 27236 individuals in our cohort died during the follow-up period, corresponding to a mortality rate of 8.7 per 10000 person-years. Within this cohort we identified 50683 with anxiety disorders and 76202 with depression (69227 (61.6\%) girls and women and $43211(38.4 \%)$ boys and men). Individuals diagnosed with anxiety disorders contributed a total of 293866 person-years of observation. Among people diagnosed with anxiety disorders, $14447(28.5 \%)$ had a comorbid diagnosis of depression. A total of 1066 people with anxiety disorders $(2.1 \%)$ died during the follow-up period, including $410(2.8 \%)$ individuals with comorbid anxiety disorders and depression (Table DS1).

\section{All-cause mortality rates in people with anxiety disorders}

The fully adjusted all-cause MRR for individuals diagnosed with anxiety disorders was 1.66 (95\% CI 1.56-1.77) compared with people from the background population without these diagnoses (reference group, Table 1). Although diagnoses of anxiety disorders were consistently associated with higher mortality rates, MRRs varied by time since first diagnosis (Table DS2). Interaction between gender and anxiety disorders seemed clinically relevant, but did not reach statistical significance for anxiety disorders $(P=0.08$, Table DS3). The MRRs were significantly elevated among individuals with anxiety disorders independent of comorbid somatic disorders (Table DS4). Allcause MRRs for people diagnosed with anxiety disorders were slightly attenuated by taking comorbid substance misuse into account (1.24, 95\% CI 1.16-1.32, Table DS5). We observed a significant dose-response relationship between the number of different anxiety disorders and mortality risk, especially in individuals with more than two anxiety disorders, who were at increased risk (Table 2).

\section{Causes of death in people with anxiety disorders}

In total, 1066 individuals diagnosed with anxiety disorders died during the follow-up period; $641(60.1 \%)$ died from natural causes and $425(39.9 \%)$ from unnatural causes. Among people diagnosed with anxiety disorders, the fully adjusted MRR was 1.39 (95\% CI 1.28-1.51) for natural causes of death and 2.46 (95\% CI 2.20-2.73) for unnatural causes of death, compared with individuals without anxiety disorders (Table 1). The MRRs for natural and unnatural causes of death did not differ between men and women (Table DS3) diagnosed with anxiety disorders. The MRRs for natural and unnatural causes of death were significantly elevated among people with anxiety disorders independent of comorbid somatic disorders (Table DS4). After taking comorbid substance misuse into account, only MRRs for unnatural causes of death were significantly elevated among individuals with anxiety disorders, whereas MRRs for natural causes fell short of significance (MRR $=1.07,95 \%$ CI $0.98-1.16$, Table DS5). The analyses exploring the effect of specific anxiety disorders did not indicate that just one specific type of anxiety disorder was driving all associations (Table 2). All specific anxiety disorders were associated with higher overall mortality rates. The risk for natural death was increased among patients 
Table 1 Mortality rate ratios of people with anxiety disorders (2002-2011)

\begin{tabular}{|c|c|c|c|c|}
\hline & \multirow[b]{2}{*}{ First adjustment $^{a}$} & \multicolumn{3}{|c|}{ Mortality rate ratio $(95 \% \mathrm{Cl})$} \\
\hline & & Second adjustment ${ }^{b}$ & Third adjustment ${ }^{\mathrm{c}}$ & Fourth adjustment $^{d}$ \\
\hline All causes & 3.71 (3.49-3.94) & $3.74(3.29-4.24)$ & $2.16(2.03-2.30)$ & $1.66(1.56-1.77)$ \\
\hline Unnatural causes & $6.47(5.86-7.14)$ & $6.85(5.74-8.60)$ & $4.63(4.17-5.13)$ & $2.46(2.20-2.73)$ \\
\hline Natural causes & $2.89(2.67-3.13)$ & $2.77(2.37-3.24)$ & $1.58(1.46-1.71)$ & $1.39(1.28-1.51)$ \\
\hline \multicolumn{5}{|c|}{$\begin{array}{l}\text { a. Mortality rate ratios derived from comparison with population controls adjusted for calendar year, age, gender and the interaction of age with gender. } \\
\text { b. Mortality rate ratios derived from comparison with sibling controls adjusted for calendar year, age, gender and the interaction of age with gender. } \\
\text { c. Mortality rate ratios derived from comparison with population controls adjusted for calendar year, age, maternal and paternal age, gender, place of residence at time of birth, } \\
\text { somatic comorbidity and the interaction of age with gender. } \\
\text { d. Mortality rate ratios derived from comparison with population controls adjusted for calendar year, age, maternal and paternal age, gender, place of residence at time } \\
\text { of birth, somatic comorbidity, depression and the interaction of age with gender. }\end{array}$} \\
\hline
\end{tabular}

with acute stress reaction, generalised anxiety disorder, panic disorder and social phobia. Specific phobia was the only anxiety disorder that did not increase the risk of dying by unnatural causes; the risk for unnatural death was more than doubled in patients with acute stress reaction, generalised anxiety disorders, posttraumatic stress disorder and social phobia. We further observed a significant dose-response relationship between the number of comorbid anxiety disorders and unnatural causes of death; this effect was less prominent for natural causes of death (Table 2).

With the exception of cancer (anxiety disorders $\mathrm{MRR}=0.88$, 95\% CI 0.73-1.03), MRRs for all specific causes of death were significantly elevated among individuals with anxiety disorders compared with the general population. The cause-specific MRRs were highest for accidents (MRR $=2.60,95 \%$ CI 2.24-3.01) among people with anxiety disorders; to enable comparability we added results for depression (Table 3). In addition, we compared patients with anxiety disorder with their unaffected siblings for all-cause mortality, natural causes and unnatural causes. All risk estimates remained raised (Table 1). We found no evidence of familial confounding for causes of death (since risk estimates were significant and of the same magnitude as for the population controls).

\section{Influence of comorbid anxiety disorders}

The MRRs of people diagnosed with comorbid anxiety disorders and depression $(\mathrm{MRR}=3.02,95 \%$ CI 2.73-3.32) were higher than the MRRs of those diagnosed with anxiety disorders alone $(\mathrm{MRR}=2.10, \quad 95 \% \quad \mathrm{CI} \quad 1.94-2.27) \quad$ or depression alone $(\mathrm{MRR}=2.57, \quad 95 \% \quad$ CI $2.45-2.70) \quad$ (Fig. 1(a)). Individuals diagnosed with comorbid anxiety disorders and depression
$(\mathrm{MRR}=11.72,95 \%$ CI 10.11-13.51) had higher MRRs for unnatural causes of death than those diagnosed with anxiety disorders alone $(\mathrm{MRR}=4.25,95 \% \mathrm{CI} 3.70-4.85)$ or depression alone (MRR $=7.87,95 \%$ CI 7.26-8.53) (Fig. 1(b)). No such effects were observed for natural causes of death (Fig. 1(c)). The MRRs for deaths caused by accidents were especially increased among people with comorbid anxiety disorders and depression (MRR $=8.02,95 \%$ CI 6.47-9.81) compared with people with only one disorder (anxiety disorder MRR $=3.28,95 \%$ CI 2.71-3.92; depression MRR $=4.38,95 \%$ CI 3.86-4.95, Fig. 1(d)). The MRRs for suicides were further strikingly high among individuals diagnosed with comorbid anxiety disorders and depression $(\mathrm{MRR}=18.32$, 95\% CI 15.21-21.86), and higher than among people with a single disorder (anxiety disorder $\mathrm{MRR}=5.69,95 \%$ CI 4.70-6.86; depression $\mathrm{MRR}=13.41,95 \%$ CI 12.10-14.84, Fig. 1(e)). The MRRs for unnatural causes and all-cause mortality were higher among people without comorbid somatic disorders and without comorbid substance misuse (Tables DS6 and DS7).

\section{Discussion}

In this nationwide prospective cohort study, we explored independent and synergistic effects of anxiety disorders and depression on various causes of mortality, carefully evaluating potential moderating effects. We report three main findings. First, the adjusted odds of all-cause mortality, which followed people diagnosed with anxiety disorders until they were 57 years old, were 1.7 compared with the general population. Around $40 \%$ of all deaths in people with anxiety disorders were from unnatural causes. Second, we found evidence that anxiety disorders constitute a significant risk factor for premature mortality independent of comorbid depression. In

Table 2 Mortality rate ratios of people with specific anxiety disorders and number of diagnoses (2002-2011)

\begin{tabular}{|c|c|c|c|}
\hline & \multicolumn{3}{|c|}{ Mortality rate ratio $(95 \% \mathrm{Cl})$} \\
\hline & All causes & Unnatural causes & Natural causes \\
\hline \multicolumn{4}{|l|}{ Anxiety diagnosis ${ }^{a}$} \\
\hline Acute stress reaction & $1.69(1.52-1.88)$ & $2.94(2.49-3.46)$ & $1.30(1.12-1.49)$ \\
\hline Agoraphobia & $1.31(1.03-1.64)$ & $1.55(1.00-2.28)$ & $1.24(0.92-1.62)$ \\
\hline Generalised anxiety disorder & $1.62(1.35-1.93)$ & $2.55(2.00-3.19)$ & $1.64(1.36-1.95)$ \\
\hline Panic disorder & $1.31(1.11-1.54)$ & $1.83(1.45-2.28)$ & $1.32(1.12-1.55)$ \\
\hline Post-traumatic stress disorder & $1.58(1.31-1.87)$ & $2.70(2.06-3.46)$ & $1.17(0.90-1.48)$ \\
\hline Social phobia & $1.50(1.20-1.84)$ & $2.19(1.70-2.77)$ & $1.51(1.21-1.85)$ \\
\hline Specific phobia & $1.50(1.09-1.99)$ & $1.76(0.94-2.96)$ & $1.40(0.96-1.95)$ \\
\hline \multicolumn{4}{|l|}{ Number of anxiety disorders ${ }^{b}$} \\
\hline 1 anxiety disorder & $2.09(1.95-2.23)$ & $4.34(3.88-4.85)$ & $1.56(1.43-1.70)$ \\
\hline 2 anxiety disorders & $2.46(2.06-2.91)$ & $6.50(4.93-8.39)$ & $1.65(1.30-2.06)$ \\
\hline$>2$ anxiety disorders & $3.27(2.33-4.42)$ & $8.85(5.18-13.69)$ & $2.18(1.39-3.22)$ \\
\hline \multicolumn{4}{|c|}{$\begin{array}{l}\text { a. Mortality rate ratios were adjusted for calendar year, age, maternal and paternal age, gender, place of residence at time of birth, somatic comorbidity, depression and the interaction } \\
\text { of age with gender. } \\
\text { b. Mortality rate ratios were adjusted for calendar year, age, maternal and paternal age, gender, place of residence at time of birth, somatic comorbidity and the interaction of age } \\
\text { with gender. }\end{array}$} \\
\hline
\end{tabular}




\begin{tabular}{|c|c|c|}
\hline Causes of death & Depression & Anxiety disorders \\
\hline \multicolumn{3}{|l|}{ Unnatural causes } \\
\hline Suicide & $11.36(10.25-12.52)$ & $2.44(2.11-2.80)$ \\
\hline Accident & 4.02 (3.57-4.51) & $2.60(2.24-3.01)$ \\
\hline Homicide & $2.57(1.30-4.62)$ & $2.34(1.02-4.67)$ \\
\hline \multicolumn{3}{|l|}{ Natural causes } \\
\hline Respiratory disease & $2.07(1.51-2.77)$ & $1.31(0.82-1.99)$ \\
\hline Cancer & $0.82(0.72-0.92)$ & $0.88(0.73-1.03)$ \\
\hline Digestive condition & $1.71(1.45-2.00)$ & $2.00(1.63-2.44)$ \\
\hline Cardiac disease & $1.75(1.49-2.06)$ & $1.50(1.20-1.86)$ \\
\hline Other disease & $1.59(1.50-1.69)$ & $1.39(1.28-1.51)$ \\
\hline
\end{tabular}

addition, sibling-control analyses revealed no familial confounding for causes of death. With the exception of suicides, the MRRs for varying causes of death were comparable for individuals diagnosed with anxiety disorders and those diagnosed with depression. Finally, we observed a significantly enhanced risk for all-cause mortality and unnatural causes of death in people diagnosed with comorbid anxiety disorders and depression compared with those with a single disorder. We hypothesised that these findings could be explained by anxious depression being a more severe form of psychiatric disorder, which might have important implications for clinical practice by underlining the need for treatment of both anxiety disorders and depression.

The MRRs for varying causes of deaths were significantly elevated among people with anxiety disorders while controlling for comorbid depression. To account for the heterogeneity of anxiety disorders, we individually assessed the impact of seven specific anxiety disorders on mortality risk. The mortality risks for specific anxiety disorders were overall rather similar, indicating that not just one specific type of anxiety disorder was driving all associations. Several specific anxiety disorders were observed to increase the risk of natural and unnatural causes of death. Acute stress reaction, generalised anxiety disorder, post-traumatic stress disorder and social phobia more than doubled the risk of dying from an unnatural cause of death. Previous studies reported several specific anxiety disorders to be associated with all-cause mortality ${ }^{17}$ as well as suicidal ideation and suicide attempts, ${ }^{27,28}$ but other causes of death have not been studied so far. In addition, we observed a higher mortality risk among individuals diagnosed with multiple anxiety disorders.

Among people with anxiety disorders, the highest MRRs were observed for deaths from unnatural causes (i.e. accidents, homicide and suicide). This pattern of high mortality from unnatural causes among people diagnosed with anxiety disorders has been previously observed. ${ }^{27,29-31}$ However, in this study we were able show for the first time that the increased mortality from unnatural causes in people with anxiety disorders could not be fully explained by comorbid depression. Similarly, Laan et al observed that the risk for suicides was enhanced among individuals with anxiety disorders but lower than in people with depression. ${ }^{18}$ Comorbidity strongly affected the suicide risk in anxiety disorders; individuals with comorbid anxiety disorders and depression were at significantly higher risk of dying by suicide than those with a single type of disorder. The risk of suicide among psychiatric patients has been studied extensively, whereas less attention has been given to the risk and prevention of accidental death, even though the latter is more common. Here, we observed the highest MRRs in people (a)

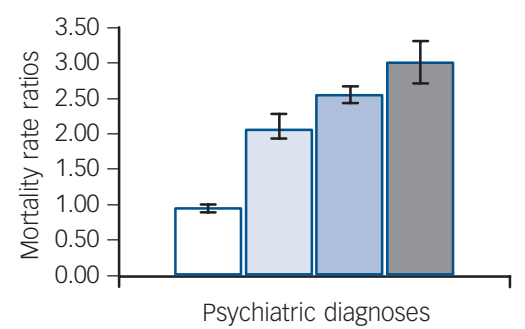

(d)

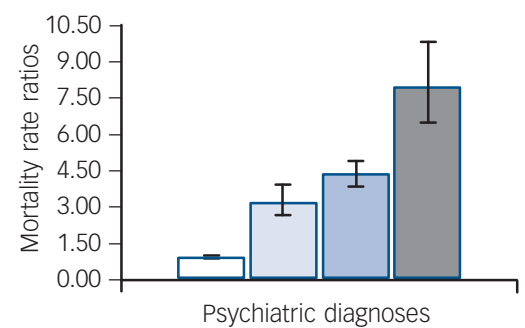

(b)

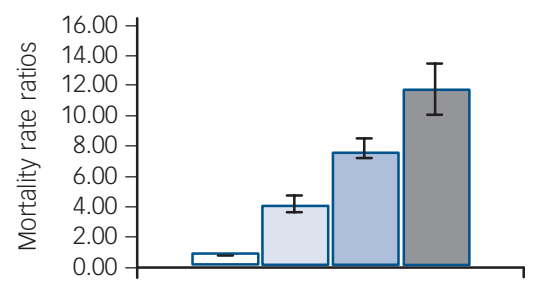

Psychiatric diagnoses

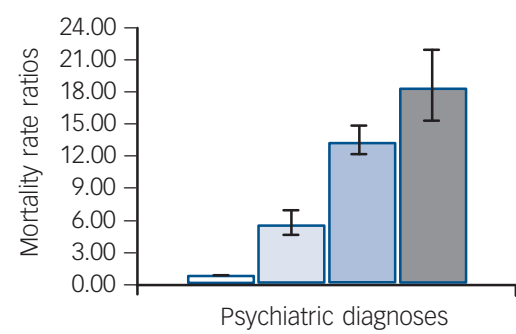

(c)

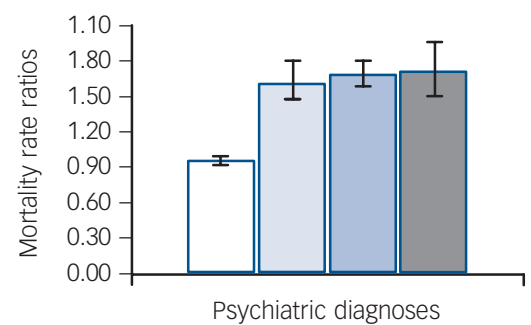

Psychiatric diagnoses
Only anxiety disorders

Only depression

Anxiety disorders and depression

Fig. 1 Influence of comorbid anxiety disorders on mortality in people with and without depression (2002-2011).

(a) All causes, (b) unnatural causes, (c) natural causes, (d) accidents and (e) suicides. Mortality rate ratios were derived from multivariate analysis and adjusted for calendar year, age, maternal and paternal age, gender, place of residence at time of birth, somatic comorbidity and the interaction of age with gender. Error bars reflect $95 \%$ confidence intervals. 
with anxiety disorders for death by accidents, most notably among those with a double diagnosis of anxiety disorders and depression. In addition, we found an increased risk of homicide among people diagnosed with anxiety disorders.

In absolute numbers, most persons diagnosed with anxiety disorders died from natural causes. Thus, the escalation of deaths from diseases and medical conditions in people with anxiety disorders is an important component of the observed excess mortality. Interestingly, comorbid depression did not result in an excess death risk from natural causes in individuals with anxiety disorders, despite the fact that most people with depression died from natural causes. Only a few mortality studies have examined gender-specific effects of anxiety disorders, providing conflicting results. ${ }^{17,32}$ In our study we found that in individuals with anxiety disorders no gender-specific effects on mortality risk could be observed.

Severe psychiatric disorders have been shown to be associated with an increased risk of somatic comorbidity ${ }^{33,34}$ and psychiatric patients might be underdiagnosed and undertreated for medical conditions. ${ }^{35}$ In addition, substance misuse is more common in anxiety disorders and depression, as depressed and anxious individuals may turn to drugs or alcohol as a form of selfmedication. ${ }^{36,37}$ In this study we could confirm mediating effects of somatic comorbidities and substance misuse on the mortality risk in people with anxiety disorders. However, in individuals with anxiety disorders mediating effects were nearly absent for unnatural causes of death. Other hazardous health styles such as physical inactivity, ${ }^{38,39}$ increased smoking rates, ${ }^{40,41}$ unhealthy eating patterns ${ }^{42-46}$ and lack of treatment seeking, ${ }^{47}$ as well as stress and biological dysregulation, ${ }^{48-50}$ might have further contributed to the observed excess mortality in people with anxiety disorders.

\section{Strengths and limitations}

We studied a nationwide population-based cohort that included all individuals born in Denmark between 1955 and 2006, with almost complete follow-up data for up to 57 years. Thus, our findings are unlikely to be explained by biases in selection of the study population or non-differentiated attrition during follow-up. There are, however, two main limitations in this study. First, we used patient registers to identify individuals diagnosed with anxiety disorders, since it was necessary to use routinely collected data to provide precision for the fairly rare outcomes investigated. Register-based diagnoses are clinical diagnoses and not the result of systematic, well-described uniform assessments. Despite a generally acceptable accuracy of anxiety disorder diagnoses in the registers, ${ }^{51}$ individuals with milder forms of anxiety disorders are less likely to present to secondary services and therefore might be underrepresented in our study. Thus, our findings might not be representative of the entire spectrum of anxiety disorders. We carefully adjusted for factors that might affect treatment seeking among people diagnosed with anxiety disorders, such as somatic and mental comorbidity. However, significant predictors of clinical outcome such as symptom severity or acceptance of the disorder might still differ between patients with or without treatment. Second, some of the causes of death may be prone to misclassification. Whereas most deaths classified as suicides reflect true suicides, it is possible that some deaths classified as accidental are in fact suicides.

\section{Implications}

This study further underlines that anxiety disorders constitute a major clinical and public health problem. Anxiety disorders were associated with a significantly increased mortality risk, and the co-occurrence of these disorders resulted in an additionally increased death risk. Because of the high prevalence of anxiety disorders, the associated excess mortality has an immense impact on public health. A recent meta-analysis indicated that approximately five million deaths worldwide are attributable to mood and anxiety disorders each year. ${ }^{52}$ For clinicians, it is also important to realise that anxiety disorders and depression are associated with increased mortality from both natural and unnatural causes, and that the assessment of physical health in these patients is always important.

\begin{abstract}
Sandra M. Meier, PhD, National Centre for Register-Based Research, Aarhus University, Aarhus, The Lundbeck Foundation Initiative for Integrative Psychiatric Research, IPSYCH, Aarhus, and Mental Health Centre for Child and Adolescent Psychiatry, Copenhagen Region, Denmark; Manuel Mattheisen, MD, The Lundbeck Foundation Initiative for Integrative Psychiatric Research, iPSYCH, Aarhus, and Department of Biomedicine, Aarhus University, Aarhus, Denmark; Ole Mors, PhD, The Lundbeck Foundation Initiative for Integrative Psychiatric Research, IPSYCH, Aarhus, and Research Department P, Aarhus University Hospital, Risskov, Denmark: Preben B. Mortensen, DrMedSc, National Centre for Register-Based Research, Aarhus University, Aarhus, and The Lundbeck Foundation Initiative for Integrative Aarhus University, Aarhus, and The Lundbeck Foundation Initiative for Integrative
Psychiatric Research, iPSYCH, Aarhus, Denmark; Thomas M. Laursen, PhD, National Centre for Register-Based Research, Aarhus University, Aarhus, and The Lundbeck Foundation Initiative for Integrative Psychiatric Research, iPSYCH, Aarhus, Denmark; Brenda W. Penninx, PhD, Department of Psychiatry and EMGO Institute for Health and Care Research, VU University Medical Center, Amsterdam, Netherlands
\end{abstract}

Correspondence: Sandra M. Meier, National Centre for Register-Based Research, Aarhus University, Fuglesangs Allé 4, 8210 Aarhus V, Denmark. Email: smeier@econ.au.dk

First received 5 Jul 2015, final revision 24 Feb 2016, accepted 27 Feb 2016

\section{Funding}

This study was supported by the Lundbeck Foundation, within the context of the Lundbeck Foundation Initiative for Integrative Psychiatric Research, iPSYCH, and Mental Health in Foundation Initiative for Integrative Psychiatric Research, IPSYCH, and Mental Health in
Primary Care (MEPRICA). S.M.M. received further funding from the Mental Health Services, Capital Region, Copenhagen, Denmark, and M.M. from the Stanley Medical Research Institute.

\section{References}

1 Pedersen CB, Mors O, Bertelsen A, Waltoft BL, Agerbo E, McGrath JJ, et al. A comprehensive nationwide study of the incidence rate and lifetime risk for treated mental disorders. JAMA Psychiatry 2014; 71: 573-81.

2 Whiteford HA, Degenhardt L, Rehm J, Baxter AJ, Ferrari AJ, Erskine HE, et al. Global burden of disease attributable to mental and substance use disorders: findings from the Global Burden of Disease Study 2010. Lancet 2013; 382: 1575-86.

3 Chang HJ, Lin HC, Lee HC, Lin CC, Pfeiffer S. Risk of mortality among depressed younger patients: a five-year follow-up study. J Affect Disord 2009; 113: 255-62.

4 Murphy JM, Burke JD Jr, Monson RR, Horton NJ, Laird NM, Lesage A, et al. Mortality associated with depression: a forty-year perspective from the Stirling County Study. Soc Psychiatry Psychiatr Epidemiol 2008; 43: 594-601.

5 Mykletun A, Bjerkeset O, Dewey M, Prince M, Overland S, Stewart R. Anxiety, depression, and cause-specific mortality: the HUNT study. Psychosom Med 2007; 69: 323-31.

6 Mykletun A, Bjerkeset O, Øverland S, Prince M, Dewey M, Stewart R. Levels of anxiety and depression as predictors of mortality: the HUNT study. Br J Psychiatry 2009; 195: 118-25.

7 Rapp MA, Gerstorf D, Helmchen H, Smith J. Depression predicts mortality in the young old, but not in the oldest old: results from the Berlin Aging Study. Am J Geriatr Psychiatry 2008; 16: 844-52.

8 Batterham PJ, Christensen $\mathrm{H}$, Mackinnon AJ. Mental health symptoms associated with morbidity, not mortality, in an elderly community sample. Soc Psychiatry Psychiatr Epidemiol 2012; 47: 79-85.

9 Herrmann C, Brand-Driehorst S, Buss U, Ruger U. Effects of anxiety and depression on 5-year mortality in 5,057 patients referred for exercise testing. J Psychosom Res 2000; 48: 455-62.

10 Holwerda TJ, Schoevers RA, Dekker J, Deeg DJ, Jonker C, Beekman AT. The relationship between generalized anxiety disorder, depression and mortality in old age. Int J Geriatr Psychiatry 2007; 22: 241-9. 
11 Wulsin LR, Vaillant GE, Wells VE. A systematic review of the mortality of depression. Psychosom Med 1999; 61: 6-17.

12 Osby U, Brandt L, Correia N, Ekbom A, Sparen P. Excess mortality in bipolar and unipolar disorder in Sweden. Arch Gen Psychiatry 2001; 58: 844-50.

13 Cuijpers P, Vogelzangs N, Twisk J, Kleiboer A, Li J, Penninx BW. Comprehensive meta-analysis of excess mortality in depression in the general community versus patients with specific illnesses. Am J Psychiatry 2014; 171: 453-62.

14 Markkula N, Härkänen T, Perälä J, Partti K, Peña S, Koskinen S, et al. Mortality in people with depressive, anxiety and alcohol use disorders in Finland. Br J Psychiatry 2012; 200: 143-9.

15 Lee WE, Wadsworth ME, Hotopf M. The protective role of trait anxiety: a longitudinal cohort study. Psychol Med 2006; 36: 345-51.

16 Denollet J, Maas K, Knottnerus A, Keyzer JJ, Pop VJ. Anxiety predicted premature all-cause and cardiovascular death in a 10-year follow-up of middle-aged women. J Clin Epidemiol 2009; 62: 452-6.

17 Carrière I, Ryan J, Norton J, Scali J, Stewart R, Ritchie K, et al. Anxiety and mortality risk in community-dwelling elderly people. Br J Psychiatry 2013; 203: 303-9.

18 Laan W, Termorshuizen F, Smeets HM, Boks MP, De Wit NJ, Geerlings MI. A comorbid anxiety disorder does not result in an excess risk of death among patients with a depressive disorder. J Affect Disord 2011; 135 : 284-91.

19 Mors O, Perto GP, Mortensen PB. The Danish Psychiatric Central Research Register. Scand J Public Health 2011; 39: 54-7.

20 Andersen TF, Madsen M, Jorgensen J, Mellemkjoer L, Olsen JH. The Danish National Hospital Register. A valuable source of data for modern health sciences. Dan Med Bull 1999; 46: 263-8.

21 World Health Organization. The ICD-10 Classification of Mental and Behavioural Disorders: Diagnostic Criteria for Research. WHO, 1993.

22 Helweg-Larsen K. The Danish Register of Causes of Death. Scand J Public Health 2011; 39: 26-9.

23 Pedersen CB. The Danish Civil Registration System. Scand J Public Health 2011; 39: 22-5.

24 Charlson ME, Pompei P, Ales KL, Mackenzie CR. A new method of classifying prognostic comorbidity in longitudinal studies: development and validation. J Chronic Dis 1987; 40: 373-83.

25 World Health Organization. International Statistical Classification of Diseases and Related Health Problems (ICD-8). WHO, 1967.

26 Kessler RC, Berglund $\mathrm{P}$, Demler $\mathrm{O}$, Jin R, Koretz D, Merikangas KR, et al. The epidemiology of major depressive disorder: results from the National Comorbidity Survey Replication (NCS-R). JAMA 2003; 289: 3095-105.

27 Sareen J, Cox BJ, Afifi TO, de Graaf R, Asmundson GJ, ten Have M, et al. Anxiety disorders and risk for suicidal ideation and suicide attempts: a population-based longitudinal study of adults. Arch Gen Psychiatry 2005; 62: 1249-57.

28 Nock MK, Hwang I, Sampson N, Kessler RC, Angermeyer M, Beautrais A, et al. Cross-national analysis of the associations among mental disorders and suicidal behavior: findings from the WHO World Mental Health Surveys. PLOS Med 2009; 6: e1000123.

29 Crump C, Sundquist K, Winkleby MA, Sundquist J. Mental disorders and vulnerability to homicidal death: Swedish nationwide cohort study. BMJ 2013 346: 5557.

30 Crump C, Sundquist K, Winkleby MA, Sundquist J. Mental disorders and risk of accidental death. Br J Psychiatry 2013; 203: 297-302.

31 Bohnert AS, Ilgen MA, Ignacio RV, Mccarthy JF, Valenstein M, Blow FC. Risk of death from accidental overdose associated with psychiatric and substance use disorders. Am J Psychiatry 2012; 169: 64-70.

32 Van Hout HP, Beekman AT, De Beurs E, Comijs H, Van Marwijk H, De Haan M, et al. Anxiety and the risk of death in older men and women. Br J Psychiatry 2004; 185: 399-404.
33 Campayo A, De Jonge P, Roy JF, Saz P, de la Cámara C, Quintanilla MA, et al. Depressive disorder and incident diabetes mellitus: the effect of characteristics of depression. Am J Psychiatry 2010; 167: 580-8.

34 Surtees PG, Wainwright NW, Luben RN, Wareham NJ, Bingham SA, Khaw KT. Depression and ischemic heart disease mortality: evidence from the EPICNorfolk United Kingdom prospective cohort study. Am J Psychiatry 2008; 165: $515-23$.

35 Laursen TM, Munk-Olsen T, Agerbo E, Gasse C, Mortensen PB. Somatic hospital contacts, invasive cardiac procedures and mortality from heart disease in patients with severe mental disorder. Arch Gen Psychiatry 2009. 66: $713-20$

36 Fergusson DM, Boden JM, Horwood L. Tests of causal links between alcohol abuse or dependence and major depression. Arch Gen Psychiatry 2009; 66: 260-6.

37 Robinson J, Sareen J, Cox BJ, Bolton JM. Role of self-medication in the development of comorbid anxiety and substance use disorders: a longitudinal investigation. Arch Gen Psychiatry 2011; 68: 800-7.

38 Whooley MA, De Jonge P, Vittinghoff $E$, et al. Depressive symptoms, health behaviors, and risk of cardiovascular events in patients with coronary heart disease. JAMA 2008; 300: 2379-88.

39 De Wit LM, Fokkema M, Van Straten A, Lamers F, Cuijpers P, Penninx BW. Depressive and anxiety disorders and the association with obesity, physical, and social activities. Depress Anxiety 2010; 27: 1057-65.

40 Dierker LC, Avenevoli S, Stolar M, Merikangas KR. Smoking and depression: an examination of mechanisms of comorbidity. Am J Psychiatry 2002; 159: 947-53.

41 Moylan S, Jacka FN, Pasco JA, Berk M. Cigarette smoking, nicotine dependence and anxiety disorders: a systematic review of population-based, epidemiological studies. BMC Med 2012; 10: 123.

42 Holahan CJ, Moos RH, Holahan CK, Cronkite RC, Randall PK. Drinking to cope and alcohol use and abuse in unipolar depression: a 10-year model. J Abnorm Psychol 2003; 112: 159-65.

43 Crum RM, Mojtabai R, Lazareck S, Bolton JM, Robinson J, Sareen J, et al. A prospective assessment of reports of drinking to self-medicate mood symptoms with the incidence and persistence of alcohol dependence. JAMA Psychiatry 2013; 70: 718-26.

44 Luppino FS, De Wit LM, Bouvy PF, Stijnen T, Cuijpers P, Penninx BW, et al Overweight, obesity, and depression: a systematic review and meta-analysis of Iongitudinal studies. Arch Gen Psychiatry 2010; 67: 220-9.

45 Forlani M, Morri M, Belvederi Murri M, Bernabei V, Moretti F, Attili T, et al. Anxiety symptoms in 74+ community-dwelling elderly: associations with physical morbidity, depression and alcohol consumption. PLOS ONE 2014; 9: e89859.

46 Mason SM, Flint AJ, Roberts AL, Agnew-Blais J, Koenen KC, Rich-Edwards JW. Posttraumatic stress disorder symptoms and food addiction in women by timing and type of trauma exposure. JAMA Psychiatry 2014; 71: 1271-8.

47 Lack D, Holt RI, Baldwin DS. Poor monitoring of physical health in patients referred to a mood disorders service. Ther Adv Psychopharmacol 2015; 5: $22-5$.

48 Vogelzangs N, Beekman AT, De Jonge P, Penninx BW. Anxiety disorders and inflammation in a large adult cohort. Transl Psychiatry 2013; 3: e249.

49 Thomas AJ, Davis S, Morris C, Jackson E, Harrison R, O'Brien JT. Increase in interleukin-1beta in late-life depression. Am J Psychiatry 2005; 162: 175-7.

50 Wium-Andersen MK, Orsted DD, Nielsen SF, Nordestgaard BG. Elevated C-reactive protein levels, psychological distress, and depression in 73, 131 individuals. JAMA Psychiatry 2013; 70: 176-84.

51 Meier S, Petersen L, Mattheisen M, Mors O, Mortensen PB, Laursen TM. Secondary depression in severe anxiety disorders: a population-based cohort study in Denmark. Lancet Psychiatry 2015; 6: 515-23.

52 Walker ER, McGee RE, Druss BG. Mortality in mental disorders and global disease burden implications: a systematic review and meta-analysis. JAMA Psychiatry 2015; 72: 334-41. 